\title{
Dados abertos: um estudo em trabalhos acadêmicos no contexto brasileiro
}

\author{
CaudioSonaglioAlbeno Doutor em Administração. Universidade Federal do Pampa (UNIPAMPA) - Brasil. \\ claudio.albano@gmail.com \\ WtterleesJr. Mestre em Administração. Universidade Federal do Pampa (UNIPAMPA) - Brasil. \\ walter.leaes@hotmail.com \\ KathianeBenedatti Carso Doutora em Administração. Universidade Federal do Pampa (UNIPAMPA) - Brasil. \\ kathianecorso@unipampa.edu.br
}

\begin{abstract}
RESUMO
0 presente artigo tem como principal proposta de contribuição apresentar um panorama dos trabalhos desenvolvidos, no contexto brasileiro, sobre o tema dados abertos e publicados em bases eletrônicas de trabalhos acadêmicos. Como suporte teórico para o estudo foram abordados os temas: bibliometria, governo aberto e dados abertos. Trata-se de um trabalho de natureza exploratória, com abordagem quantitativa, desenvolvido por intermédio de uma pesquisa bibliográfica. 0 ambiente da pesquisa, representado pelas bases de dados consultadas foram orientadas pela classificação Qualis, onde o recorte foi nos periódicos classificados como A1, A2, B1 e B2, nas áreas de Ciências Políticas, Administração e Ciências Sociais Aplicadas, bem como a plataforma Spell. Foram encontrados 17 artigos relacionados ao tema dados abertos, distribuído em 14 periódicos. Constatou-se que o tema é recente no cenário acadêmico brasileiro, a maior parte dos trabalhos tem foco no segmento governo. Para apresentar um panorama dos trabalhos foram identificados os autores que publicam trabalhos, respectivas instituições, periódicos que publicam e contexto no qual os trabalhos foram desenvolvidos.
\end{abstract}

Palavras-chaves: Bibliometria. Dados abertos. Produção acadêmica.

\section{Open data: a study in academic works in the brazilian context}

\begin{abstract}
The present article has as main contribution proposal to present an overview of the work developed, in the Brazilian context, on the subject open data and published in electronic databases of academic works. As a theoretical support for the study, the following topics were addressed: bibliometrics, open government and open data. It is a work of exploratory nature with a quantitative approach, developed through a bibliographical research. The research environment, represented by the databases consulted, was oriented by the Qualis classification, where the cut was in the periodicals classified as A1, A2, B1 and B2, in the areas of Political Science, Administration and Applied Social Sciences, as well as the Spell platform.The 17 articles found related to open data, were distributed in 14 journals. It was found that the theme is recent in the Brazilian academic scenario, most of the work is focused on the government segment. In order to present an overview of the works, the authors that publish papers, their institutions, the periodicals they publish and the context in which the works are developed were identified.
\end{abstract}

Keywords: Academic production. Bibliometr. Open data. 


\section{INTRODUÇÃO EJUSTIFICATIVA}

As organizações públicas estão, cada vez mais, sendo cobradas por parte da sociedade, que demanda melhor qualidade na prestação de serviços por parte destas. Objetivando o efetivo monitoramento das organizações públicas pela sociedade, uma das exigências está relacionada com questões de transparência na gestão dos recursos públicos. Nesse contexto, as tecnologias de informação e comunicação (TICs) se inserem como possível ferramenta para auxiliar a atender estas demandas (FREITAS; DACORSO, 2014).

Nas duas últimas décadas ocorre um debate na administração pública sobre o impacto que acarretaria a adoção de tecnologias de informação e comunicação (TICs) em organizações públicas. De acordo com Braga e Gomes (2015) estas sofreram diversas alterações desde que os agentes públicos passaram a utilizar as TICs para melhorar seus programas e serviços.

Atualmente, com a utilização das diversas plataformas de TICs pelos governos, emergiu o termo Governo 2.0, visto como o governo na "era da informação" (HARRISON; PARDO; COOK, 2012). Para estes autores com o advento da Web 2.0, consumidores de informações também são produtores de informações, havendo troca de informações entre usuários e organizações; este intercâmbio de informações intensifica a relação da sociedade com seus governos.

A adoção das TICs por parte dos governos ocasionou a melhora no relacionamento entre o governo e cidadão. A elevação desta relação a outro patamar possibilitou um novo modelo de governo, o governo aberto. A ideia de governo aberto é oriunda de uma nova estratégia de abertura de informações por parte dos governos, que visam aumentar a interação com a sociedade e melhorar os resultados de suas atividades (ALBANO; REINHARD, 2015).

O símbolo do governo aberto é a Declaração de Governo Aberto (BRASIL, 2011), entre os compromissos assumidos pelos países que aderiram a este acordo, está o aumento da participação da sociedade por meio da disponibilização e abertura de dados governamentais. Neste sentido, surge a responsabilidade do governo em disponibilizar os dados governamentais em formato aberto.

De acordo com Hellberg (2014), Dados Governamentais Abertos tratam da disponibilização dos dados das instituições governamentais em formato aberto, passível de reutilização. Este autor aborda que, em uma perspectiva global, a abertura de dados públicos pode levar a uma ascensão econômica de quarenta bilhões de euros por ano na União Europeia. Ainda segundo este autor, além dos benefícios econômicos, esta iniciativa é um fator-chave na promoção da transparência, na prestação de contas do governo, uma vez que irá promover a participação dos cidadãos na vida política e social. A World Wide Web Consortium aborda como um dos pontos mais relevantes, para aderir aos dados governamentais, a possibilidade deste gerar novos serviços que favoreçam a sociedade (W3C, 2011).

O tema dados abertos é citado frequentemente como um tema novo, precoce e que necessita de mais trabalhos para que efetivamente se possam verificar todas as suas potencialidades, (ALBANO, 2014; KLEIN; LUCIANO; MACADAR, 2015; FREITAS; DACORSO, 2014). De outra parte autores como Robinson, Zeller e Yu (2009), Gurstein (2011) e Prince, Jolías e Brys (2013), afirmam para que dados abertos efetivamente sejam implementados é necessário a formação de redes entre governos e sociedade. Robinson, Zeller e Yu (2009) vão um pouco além e dizem que os governos deveriam designar boa parte das tarefas a serem realizadas com dados abertos para organizações da sociedade, pois estas teriam maior capacidade e dinamismo para executar algumas das tarefas necessárias à efetividade deste contexto.

A partir do exposto até aqui, este trabalho apresenta a seguinte questão de pesquisa: No contexto acadêmico brasileiro, qual ou quais são as abordagens prevalecentes em relação à temática de dados abertos?

0 trabalho apresenta ainda os seguintes objetivos: identificar quais autores publicam trabalhos relacionados ao tema; relacionar em quais periódicos/eventos/base de dados em que estes trabalhos foram publicados; e finalmente verificar se é possível confirmar (ou não) a precocidade do tema no contexto brasileiro. Acredita-se que ao atender a questão de pesquisa e objetivos do trabalho, o mesmo possa realizar uma contribuição para o tema, qual seja de apresentar um panorama dos trabalhos desenvolvidos, no contexto brasileiro, sobre o tema dados abertos e publicados em bases eletrônicas de trabalhos acadêmicos. 
O trabalho está estruturado da seguinte forma: na próxima seção apresenta-se o referencial teórico, onde são abordados: bibliometria, governo aberto e dados abertos. A seguir descrevem-se os procedimentos metodológicos utilizados para o desenvolvimento do trabalho, e após as considerações finais, limitações do trabalho e sugestões para trabalhos futuros.

\section{REFERENCIAL TEÓRICO}

Neste tópico, buscou-se expor a importância dos estudos bibliométricos, evidenciar seus conceitos e objetivos, o portunizando a base para desenvolver a análise bibliométrica dos dados utilizados neste trabalho. Também é abordado o tema governo aberto, em uma breve relação com governo eletrônico e também com dados abertos, posteriormente são apontadas as novas relações entre sociedade e governo, decorrentes das possibilidades da adoção de governo aberto. No último tópico, dados abertos, conceitua-se o tema descrevendo sua origem, potenciais benefícios e vantagens de sua adoção, bem como sua importância no cenário da sociedade atual.

\subsection{Bibliometria}

Os avanços da ciência ocorrem por intermédio de diversas formas, entre estas se pode citar os estudos reflexivos, os quais permitem a contemplação do que foi produzido pela comunidade científica (SPLITTER; ROSA; BORBA, 2012). No entendimento de Splitter e Rosa (2012), a Bibliometria surge a partir do desenvolvimento da ciência, à medida que se fez necessário avaliar e acompanhar a ampliação e os avanços obtidos pelas diversas áreas do conhecimento.

Os primeiros estudos bibliométricos foram produzidos por J ames Mc Keen Cattel (1906), Coles e Eales (1917) e Hulme (1923), entretanto estes autores ainda não utilizavam o termo (SPLITTER; ROSA; BORBA, 2012). Nos últimos anos percebe-se o crescimento dos estudos desta natureza, como exemplo citamos o evento Encontro Nacional dos Programas de Pós-Graduação em Administração (EnANPAD). No ano de 2005, trabalhos bibliométricos representavam apenas $1 \%$ das publicações no evento, enquanto em 2010, este percentual já era de aproximadamente $4 \%$.

A Bibliometria é para Guedes e Borschiver (2005) uma ferramenta estatística, que, por intermédio de indicadores, realiza o tratamento e a gestão da informação, do conhecimento e da produtividade, necessária ao planejamento, avaliação e gestão da ciência de uma determinada comunidade científica. A Bibliometria se propõe a criar indicadores de avaliação da produção científica por meio de ferramentas matemáticas e estatísticas (SPLITTER; ROSA; BORBA, 2012) visando mensurar o conhecimento sobre a produção do mesmo, possibilitando gerar novos conhecimentos e novos enfoques.

Neste sentido, a Bibliometria torna-se um recurso importante para o estudo dos acontecimentos, para difusão, consolidação, compreensão, debate, análise e repercussão do conhecimento, para medir a influência de pesquisadores ou periódicos e traçar seus perfis.

Para Amaral et al. (2004), os indicadores bibliométricos são classificados em indicadores de uma dimensão e indicadores de duas dimensões denominados indicadores de ligação. Nos indicadores de uma dimensão, 0 autor subdivide em indicadores de atividades (contagem de publicações) e indicadores de impacto (contagem de citações recebidas). Para este autor, os indicadores de ligação são usados para a criação de mapas descritivos, que podem ter como foco redes de relacionamento entre pesquisadores, instituições e países; sua importância decorre da possibilidade de visualizar as redes de colaboração e para identificar a ligação entre temas ou áreas de pesquisa.

Existem diversas leis e princípios quando se aborda o tema bibliometria. Para fins deste trabalho vamos destacar as leis de Lotka e Zipf. A segunda faz referência à frequência com que determinadas palavras aparecem nos trabalhos relacionados a determinado tema (SPLITTER; ROSA, 2012; SPLITTER; ROSA; BORBA, 2012). A lei de Lotka, preocupa-se com a produtividade dos autores, com relação a determinado tema. Segundo esta lei alguns autores (poucos) publicam muito e outros autores (muitos) publicam pouco sobre determinado tema.

Finalmente, entende-se que a bibliometria é adequada para este trabalho, pois segundo Splitter e Rosa (2012), por intermédio de estudos bibliométricos pode-se identificar: o crescimento, e até mesmo o 
envelhecimento, de um campo da ciência; a evolução cronológica da produção científica; a produtividade de autores e instituições; colaboração entre pesquisadores e instituições; entre outros aspectos.

\subsection{Governo Aberto}

No Brasil, apesar do governo utilizar recursos da TIC desde a década de 1950, o termo governo eletrônico começa a ser empregado apenas em meados da década de 1990. Porém, o uso de TICs na prestação de serviços pelo governo ao cidadão ganhou evidência com a entrega da Declaração de Imposto de Renda, disponibilizado em 1998 (MORAES; MEIRELES, 2014). 0 conceito de governo eletrônico segundo a ONU (2004) é a utilização das TIC e sua aplicação pelo governo para a prestação de informações e serviços públicos para as pessoas.

A adoção do governo eletrônico possibilitou uma nova perspectiva na integração entre os cidadãos e o governo, esta nova relação é chamada de governo aberto. De acordo com Lathrop e Ruma (2010), governo aberto significa governo onde o cidadão não quer apenas o acesso à informação, documentos e processos, mas também participar de uma forma significativa. Para Harrison, Pardo e Cook (2012), o governo aberto, por meios das TICs, fortalece a colaboração entre sociedade e governo e aumenta transparência dos governos.

O marco fundamental do governo aberto foi a Declaração de Governo Aberto (BRASIL, 2011). Segundo esta declaração governo aberto possui como compromissos básicos aumentar a disponibilidade de informações sobre as atividades governamentais, apoiar a participação cívica, implementar os mais altos padrões de integridade profissional por toda a administração e ampliar o acesso a novas tecnologias para fins de abertura e prestação de contas.

É imprescindível distinguir o conceito de governo eletrônico e governo aberto. 0 governo eletrônico faz referência a aplicação das TIC para os processos administrativos, no sentido de aumentar a oferta de serviços administrativos (a sociedade) pelo governo. Todavia, governo aberto, que surgiu em um segundo momento, tem a intenção de alterar a relação entre o governo e o cidadão, aperfeiçoar a administração pública através do diálogo constante com a sociedade, identificando suas necessidades e auxiliando na tomada de decisões em prol do coletivo (OLIVERIO, 2011).

Harrison, Pardo e Cook (2012) entendem que as ideias de abertura do governo estão alastrando-se ao redor do mundo. Para estes autores, governos como os da Austrália, Grã-Bretanha e Canadá estão buscando a abertura de seus governos por meio das TICs, disponibilizando seus dados em formato aberto para o cidadão. De forma coerente a este pensamento Parycek, Hochtl e Ginner (2014), defendem que, para o governo ser um sistema aberto que interage e coopera com o seu ambiente, é necessário que este disponibilize seus dados em formato aberto. Este fato reforça a premissa de que a adoção de dados abertos, talvez seja uma das principais oportunidades dentro do leque de novos serviços proporcionados pela plataforma de governo aberto (ALBANO, 2014).

\subsection{Dados Abertos}

J ohnson (2014) aborda dados abertos (públicos ou privados) são dados disponíveis publicamente, em formato legível por máquinas, livre de licenças e com menor nível de granularidade possível. Para este autor, o movimento tem se destacado como política pública, principalmente, em razão da adoção por parte dos governos. A adoção e disseminação das plataformas de e-gove governo aberto, aliado a evolução das TICs, originaram grandes bases de dados sob o domínio dos governos. Estes ideários permitiram que a sociedade, cada vez mais usuária de TICs, venha a ter acesso às bases de dados mantidas pelos governos, esta interação impulsionou a adoção de dados governamentais abertos.

Para Clabo e Ramos-Vielba (2015) Dados Governamentais Abertos (DGA) pode ser definido como as informações do setor público, que são compartilhadas com a sociedade em formato aberto através da Internet, de forma que promova a sua análise e reutilização. De acordo com estes autores DGA pode ser visto como uma filosofia que sustenta a democratização da informação. De forma mais sucinta Sapena, Peset e BEnavent (2011), define DGA como dados públicos apresentados em formato padrão aberto, de modo que eles possam ser usados para fornecer serviços e aplicações úteis para os usuários, podendo ser consumida de dispositivos móveis ou fixos. 
Conforme o entendimento de Albano (2014), a iniciativa de DGA surgiu, em um primeiro momento, na Europa Ocidental e América do Norte, principalmente com a instituição do portal www.data.gov pelos Estados Unidos e www.data.gov.uk pela Inglaterra. Já o Brasil lançou em 2010 seu portal de dados abertos (www.dados.gov.br). De acordo com o próprio Portal, é a ferramenta pela qual o governo disponibiliza para todos encontrarem e utilizarem dados e informações públicas, buscando ser o interlocutor entre a sociedade e o governo. Ainda segundo este autor, o principal marco na legislação brasileira sobre o tema foi a Lei 15.527/2011 - Lei de Acesso à Informação.

De acordo com Janssen, Charalabidis e Zuiderwijk (2012) é pressuposto básico que a adoção de DGA pode gerar benefícios. Segundo estes autores pode-se agrupar estes benefícios em três áreas: política e sociais, econômica e operacional e técnica. $\mathrm{Na}$ área econômica são apontados como benefícios, dentre outros, $\mathrm{O}$ incentivo à inovação, desenvolvimento de novos produtos e serviços e disponibilidade de informação para investidores e empresas.

Ainda para Janssen, Charalabidis e Zuiderwijk (2012), colocam como benefício político e social desta iniciativa o aumento da transparência e prestação de contas dos governos, fortalecendo a confiança e satisfação do cidadão. Estes autores definem como um dos principais benefícios o aproveitamento da inteligência coletiva da sociedade, possibilitando tomadas de decisões coletivas, melhores que isoladas. Outro ponto relevante é a possibilidade dos DGA auxiliarem nos processos de formulação de políticas e serviços sociais inovadores.

Parycek, Hochtl e Ginner (2014) em pesquisa sobre a implementação de DGA na cidade de Viena, observaram como principais benefícios decorrentes desta: o melhor processamento de dados, aumento da motivação dos empregados, melhoria da imagem do setor pública, aumento da eficiência, rápida disponibilização de dados, aumento da participação do cidadão e legitimação de políticas.

Para autores como McKinsey (2013), Ubaldi (2013) e Santos Neto et al. (2013), apesar dos benefícios existem muitos desafios a serem superados por esta inciativa. São necessários estudos que possibilitem a criação de mecanismos para a integração e interoperabilidade de dados, visando sua interligação e reutilização, bem como estudos visando explorar seu potencial econômico.

\section{PROCEDIMENTOS METODOLÓGICOS}

Este estudo, com características exploratórias foi desenvolvido em um cenário em que encontramos poucos trabalhos, segundo Gil (2008) estudos exploratórios são realizados em áreas com pouco conhecimento publicado e sistematizado. A abordagem quantitativa é pressuposto dos estudos bibliométricos, de acordo com Guedes e Borschiver (2005), estes estudos devem utilizar ferramentas estatísticas para realizar a gestão da informação de determinada área de estudo. Em relação ao método de investigação o trabalho caracteriza-se por ser um estudo bibliográfico (MIGUEL, 2012).

0 delineamento da pesquisa considera 0 ambiente da mesma, aqui representado pelas bases de dados consultadas. Estas bases foram orientadas pela classificação Qualis do ano de 2014, o recorte foi nas bases de dados dos periódicos classificados como A1, A2, B1 e B2, nas áreas de Ciências Políticas, Administração e Ciências Sociais Aplicadas. A escolha destas áreas é justificada, pois segundo al guns autores como Angélico (2012) e Deloitte (2012), estas áreas tem possibilidades de maior interesse pelo tema.

Uma vez identificada a área de administração como principal fonte das publicações, a busca foi estendida, com os mesmos critérios (palavras chaves utilizadas para a busca), a plataforma Spell, em razão de esta concentrar mais de 35 mil trabalhos acadêmicos relacionados à produção científica das áreas de Administração, Contabilidade e Turismo.

Para os procedimentos de busca de trabalhos, tanto nos periódicos como na base Spell, foram utilizados os termos: "dado aberto", "governo aberto", "dados governamentais abertos", "dados abertos governamentais", "dados linkados", "dados ligados", "governo" e finalmente "abertos", compreendendo título, resumo e palavras- chave. Foram consideradas publicações entre o período entre janeiro de 2006 e maio de 2016. 
Quanto à compilação dos artigos, em razão da plataforma SPELL remeter aos periódicos nos quais os artigos foram publicados, alguns artigos encontrados nesta plataforma já haviam sido localizados na pesquisa nos próprios periódicos. Sendo assim, foram mantidos apenas os resultados não repetidos.

\section{RESULTADOS}

Nesta seção do trabalho, são apresentados os resultados e respectivas análises. Foram encontrados dezessete (17) trabalhos, que satisfizeram os critérios utilizados para a busca (conforme descrito na seção procedimentos metodológicos) estes estão no Quadro 01.

Quadro 01 - Resultado das buscas

\begin{tabular}{|c|c|}
\hline Plataforma/Área & Título trabalho \\
\hline \multirow{3}{*}{ Ciências Políticas } & Lei de acesso à informação: impactos efetivos na Câmara dos Deputados. \\
\hline & $\begin{array}{l}\text { Inovação aberta na gestão pública: análise do plano de ação brasileiro para a Open } \\
\text { Government Partnership. }\end{array}$ \\
\hline & $\begin{array}{l}\text { A publicação de dados governamentais abertos: proposta de revisão da classe } \\
\text { sobre Previdência Social do Vocabulário Controlado do Governo Eletrônico. }\end{array}$ \\
\hline \multirow{8}{*}{ Administração } & Acesso à Informação: notas de pesquisa. \\
\hline & Grau de transparência de dados abertos governamentais do site dados.rs.gov.br \\
\hline & $\begin{array}{l}\text { Lições aprendidas com a utilização de dados orçamentários em formato aberto: } \\
\text { um estudo exploratório no ecossistema brasileiro. }\end{array}$ \\
\hline & $\begin{array}{l}\text { Desafios para Governos e Sociedade no Ecossistema Brasileiro de Dados } \\
\text { Governamentais Abertos. }\end{array}$ \\
\hline & $\begin{array}{l}\text { Uma abordagem para captura de informações sobre aplicações que fazem uso de } \\
\text { dados abertos. }\end{array}$ \\
\hline & IGOV: um sistema de integração de dados governamentais. \\
\hline & $\begin{array}{l}\text { Busca Contextualizada Enriquecida com Dados Abertos para Apoiar a } \\
\text { Aprendizagem Colaborativa em Redes Sociais. }\end{array}$ \\
\hline & $\begin{array}{l}\text { Tecnologias de dados abertos para interligar bibliotecas, arquivos e museus: um } \\
\text { caso machadiano. }\end{array}$ \\
\hline \multirow{2}{*}{$\begin{array}{l}\text { Ciências Sociais } \\
\text { Aplicadas }\end{array}$} & Comunicação científica e as tecnologias de informação e comunicação. \\
\hline & O E-gov como política de comunicação na gestão pública do ABC Paulista. \\
\hline \multirow{4}{*}{ SPELL } & SCiAl: Usando Dados Públicos para Agrupar Cidades Alagoanas. \\
\hline & $\begin{array}{l}\text { Aumentando a transparência do governo por meio da transformação de dados } \\
\text { governamentais abertos em dados ligados. }\end{array}$ \\
\hline & $\begin{array}{l}\text { Transparência como elemento fundamental em governo eletrônico: uma } \\
\text { abordagem institucional. }\end{array}$ \\
\hline & $\begin{array}{l}\text { Ranking das prefeituras da região Sul do Brasil: uma avaliação a partir de critérios } \\
\text { estabelecidos na Lei de Acesso à Informação. }\end{array}$ \\
\hline
\end{tabular}

Fonte: Elaborado pelos autores (2018).

O Quadro 01 apresenta a evolução quantitativa da produção científica sobre dados abertos e os periódicos nos quais foram localizadas as publicações.

Quadro 01 - Evolução da produção sobre dados abertos

\begin{tabular}{|c|c|c|}
\hline Ano & Quantitativo de Artigos & Periódicos \\
\hline \multirow{2}{*}{2011} & \multirow[t]{2}{*}{ 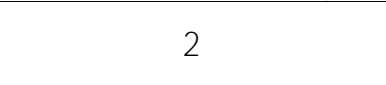 } & Comunicação \& Sociedade \\
\hline & & Revista Eletrônica de Sistemas de Informação \\
\hline \multirow{3}{*}{2013} & \multirow{3}{*}{3} & Informação \& Informação (UEL. Online) \\
\hline & & Revista Brasileira de Informática na Educação \\
\hline & & Transinformação \\
\hline \multirow[b]{2}{*}{2014} & \multirow[b]{2}{*}{4} & Cadernos da Escola do Legislativo \\
\hline & & $\begin{array}{l}\text { Cadernos de Pesquisa (Fundação Carlos Chagas. } \\
\text { Impresso) }\end{array}$ \\
\hline
\end{tabular}




\begin{tabular}{|c|c|c|}
\hline & & Revista Brasileira de Administração Científica \\
\hline \multirow{7}{*}{2015} & \multirow{7}{*}{7} & Cadernos de Pesquisa (Fundação Carlos Chagas \\
\hline & & Cadernos Gestão Pública e Cidadania \\
\hline & & Revista Economia \& Gestão \\
\hline & & Revista de Gestão e Projetos \\
\hline & & Comunicação \& Sociedade \\
\hline & & Revista Gestão \& Tecnologia \\
\hline & & Revista de Administração Pública \\
\hline 2016 & 1 & $\begin{array}{l}\text { GESTÃO.Org - Revista Eletrônica de Gestão } \\
\text { Organizacional }\end{array}$ \\
\hline
\end{tabular}

Fonte: Elaborado pelos autores (2018).

É possível perceber, pelos dados do Quadro 01, a evolução na produção de trabalhos que abordam dados abertos. Pode-se concluir que a produção está concentrada no ano de 2015 , o qual detém $41 \%$ dos trabalhos. Cabe aqui destacar que o levantamento ocorreu no primeiro semestre do ano de 2016, o que pode justificar o número baixo de publicações neste ano (2016), apenas um trabalho. Ainda sobre este Quadro, verifica-se o crescimento deste campo de estudos, à medida que houve crescimento significativo da produção de artigos nos últimos cinco anos.

Entre os periódicos que realizaram publicação sobre o tema, destacam-se três periódicos por reunir a maior parte das publicações: Cadernos de Pesquisa (Fundação Carlos Chagas), Revista Brasileira de Administração Científica e Comunicação \& Sociedade. Estes periódicos concentram $35 \%$ dos trabalhos com o tema.

Quanto aos autores e respectivas instituições, o Quadro 02 apresenta este perfil e o número de publicações. Para definir o vínculo dos autores com as instituições, foram utilizadas as informações constantes na plataforma Lattes (currículo lattes de cada autor).

Quadro 02 - Autores e Instituições

\begin{tabular}{|c|l|c|}
\hline Instituição & \multicolumn{1}{|c|}{ Autores } & $\begin{array}{c}\text { Participação } \\
\text { em Artigos }\end{array}$ \\
\hline UNIRIO & $\begin{array}{l}\text { Durval Vieira Pereira, Cláudio José Silva Ribeiro, Eduardo Fritzen, Leila } \\
\text { Cristina de Vasconcelos Andrade, Sean Wolfgand Matsui Siqueira }\end{array}$ & 2 \\
\hline UNIPAMPA & Claudio Sonaglio Albano & 2 \\
\hline PUCRS & $\begin{array}{l}\text { Marie Anne Macadar, Cristiano, Rodrigo Hickmann Klein, Ramos } \\
\text { Moreira, Edimara Mezzomo Luciano, J orge Lheureux de Freitas }\end{array}$ & 2 \\
\hline UFAL & $\begin{array}{l}\text { Fábio J osé Coutinho da Silva, João Paulo Clarindo dos Santos, Luciana } \\
\text { Campos da Costa, Ricardo Alexandre Afonso }\end{array}$ & 2 \\
\hline UFSCar & Alexandre Álvaro & 1 \\
\hline UFJF & Jairo Francisco de Souza, Lucas de Ramos Araújo & 1 \\
\hline UFR & Nilson Barbosa & 1 \\
\hline UNESC & Marciele Berger Bernardes & 2 \\
\hline UNILEGIS & $\begin{array}{l}\text { Raphael Silva Cavalcante, Roberto Campos da Rocha Miranda, Robson } \\
\text { Luiz Fialho Coutinho, Terezinha Elisabeth da Silva, Thiago Eirão Gomes }\end{array}$ & 2 \\
\hline USP & Gisele da Silva Craveiro, Nicolau Reinhard & 1 \\
\hline UFS & Antonio Luiz Rocha Dacorso, Rony Klay Viana de Freitas \\
\hline UFF & $\begin{array}{l}\text { Antonio Laurindo dos Santos Neto, Carlos Henrique Marcondes, Eliana } \\
\text { Rosa da Fonseca, lara Vidal Pereira de Souza, Rosana Portugal Tavares } \\
\text { de Moraes, Sergio de Castro Martins }\end{array}$ & 2 \\
\hline UFPE & $\begin{array}{l}\text { Bernadette Farias Lóscio, Lairson E. Rodrigues de Alencar Oliveira, } \\
\text { Vinicius Cardoso Garcia }\end{array}$ & 1 \\
\hline UNIBAN & Robson Luiz Gisoldi & 1 \\
\hline USCS & Arquimedes Pessoni \\
\hline UFSC & $\begin{array}{l}\text { Aires José Rover, Fernanda Schweitzer, Gregório Jean VarvakisRados, } \\
\text { Paloma Maria Santos, Rosângela Schwarz Rodrigues }\end{array}$ & 2 \\
\hline
\end{tabular}

Fonte: Elaborado pelos autores (2018). 
É possível constatar o total de 45 autores, destaca-se que Albano, Macadar e Pereira são os autores com mais artigos publicados com relação ao tema dados abertos. Outro aspecto importante neste contexto é a confirmação da Lei de Lotka. De acordo com Splitter, Rosa e Borba (2012), esta Lei é sintetizada pela ideia que poucos autores produzem muito e muitos autores produzem pouco, sobre determinado tema. Neste sentido, percebe-se a participação destes autores, acima citados, em $35 \%$ dos artigos analisados. Cabe aqui avaliar que, apesar deste número não representar a maioria dos artigos, trata-se de um campo de pesquisa em evolução. Outro ponto que deve ser observado é o grande número de autores com apenas um artigo sobre o tema.

Deve-se destacar, ainda sobre os principais autores, que estes não realizaram publicações em coautoria. No tocante ao quantitativo dos pesquisadores observa-se a predominância de dois autores nos artigos levantados, entretanto, existem publicações com quatro (4) e até oito (8) autores. Não foi identificado nenhum trabalho com um único autor.

A figura 01 apresenta a rede de cooperação formada pelos autores. 0 laço entre os autores representa a coautoria. 0 tamanho dos círculos é proporcional à quantidade de ligações entre os coautores. Assim, quanto maior o número de coautores diferentes, maior será o círculo do autor.

Figura 01 - Rede de autores

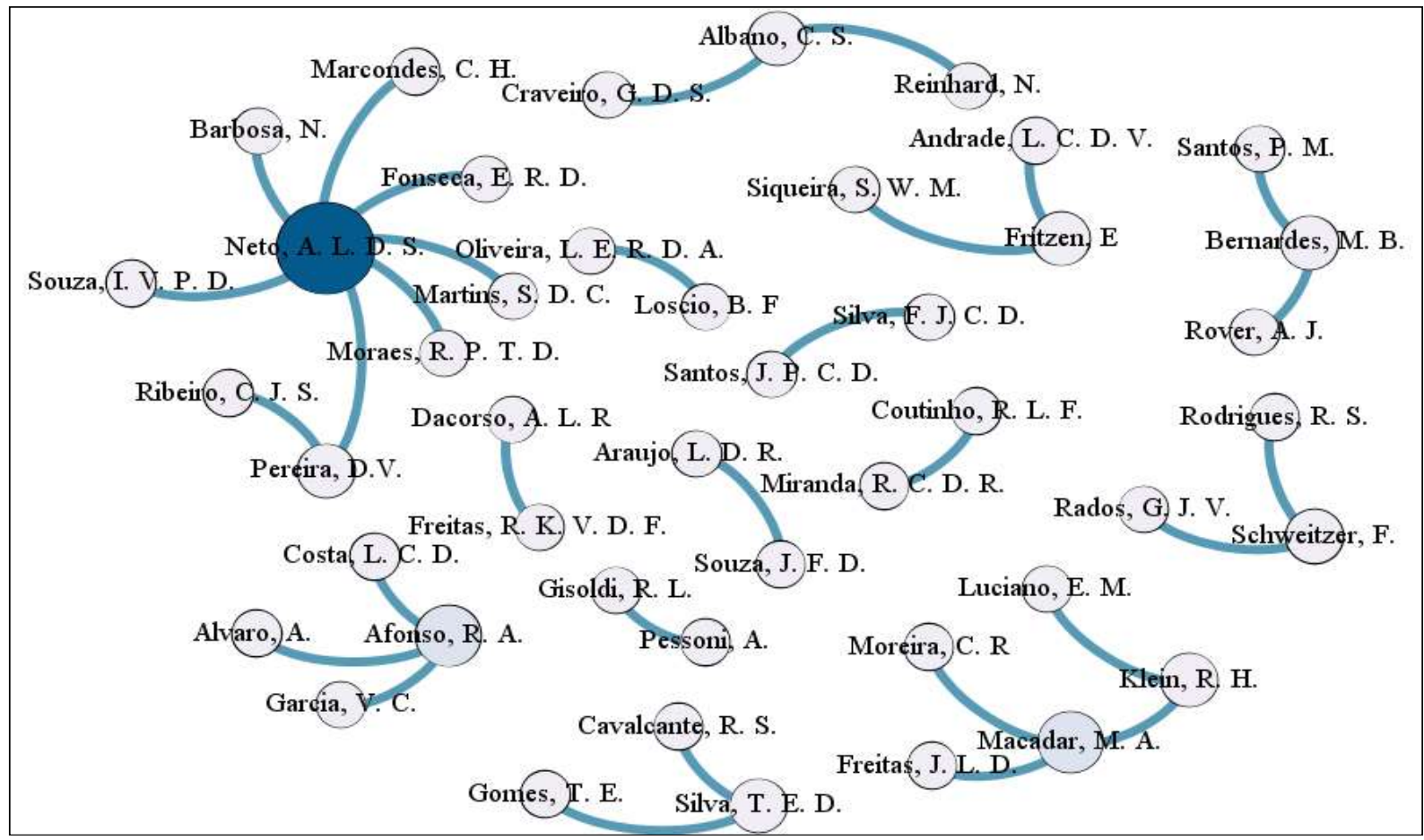

Fonte: Elaborado pelos autores (2018).

A rede de autores, ilustrada pela figura 01 , percebe-se um destaque para os autores Neto, Macadar e Afonso. Estes autores foram os que apresentaram o maior número de ligação com outros autores. A dispersão dos autores dentro da rede decorre da sua baixa densidade. Em razão da densidade da rede, pode-se perceber uma baixa interação entre os autores.

Para verificar a cooperação das Instituições de Ensino Superior (IES), no que diz respeito à produção cientifica, a figura 02 contempla a rede formada pelas 16 instituições identificadas na coleta de dados. Foi possível dimensionar esta cooperação entre as instituições pelo vinculo institucional dos autores e coautores. 0 tamanho do círculo, que representa cada entidade, é oriundo das relações entre os autores e coautores. 


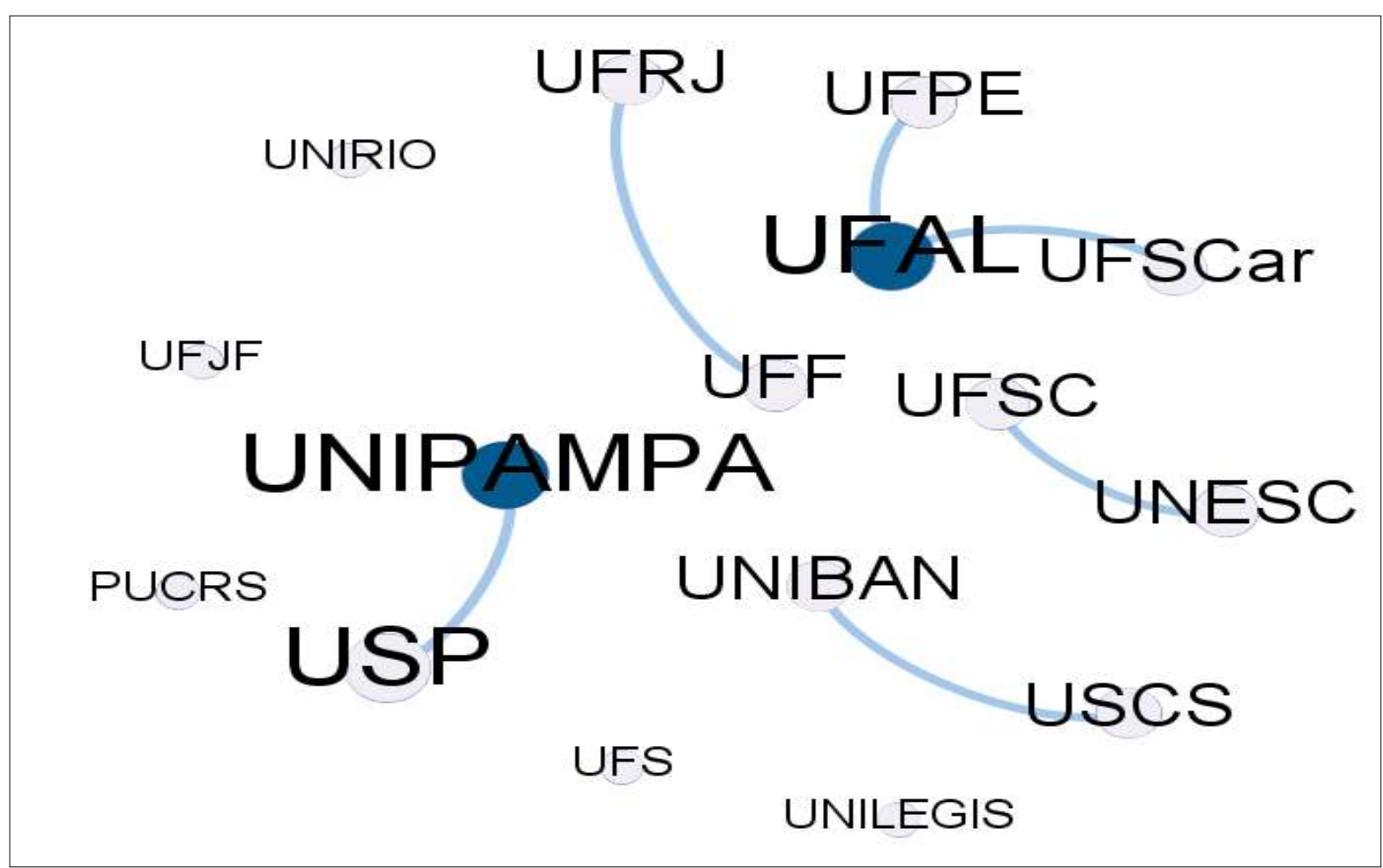

Fonte: Elaborado pelos Autores (2018).

A rede de cooperação entre as IES apresenta 16 nós e 7 arestas. Observa-se que 31\% das instituições produziram de forma isolada suas pesquisas, sem haver cooperação com outra entidade, a rede apresenta índice de densidade igual a 0,029. Este resultado representa que 1,29\% das interações possíveis entre estas instituições estão ocorrendo. Em destaque na figura, as IES que realizaram maior número de interações foram a UNIPAMPA e a UFAL. Assim como na rede de autores, estes dados demonstram a oportunidade de aumentar a cooperação entre instituições e autores. A figura 03 demonstra as palavras-chave utilizadas nos trabalhos selecionados. Na figura aparecem em evidência as seguintes palavras: dados, governo, abertos, informação e eletrônico.

Figura 03 - Frequência das Palavras-Chave

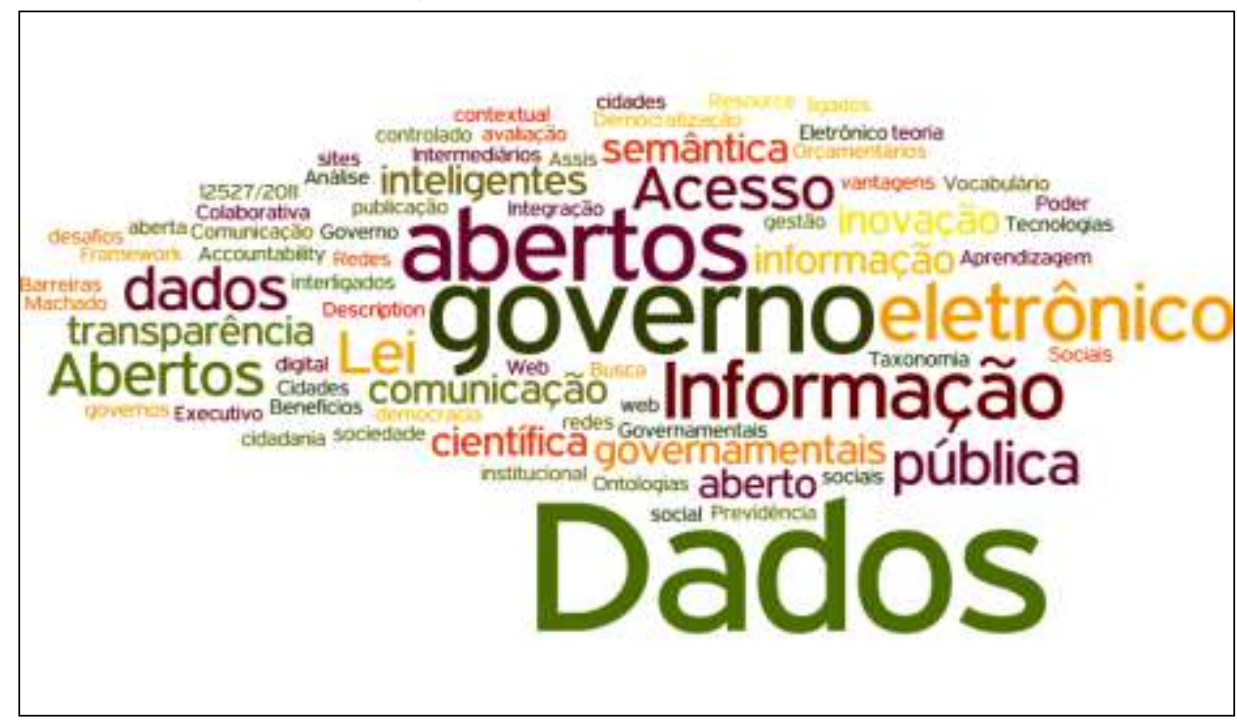

Fonte: Elaborado pelos autores (2018). 
O resultado da frequência de palavras-chave, ilustrado na figura 03, vai ao encontro da Lei de Zipf. De acordo com Splitter e Rosa (2012), esta lei define que determinadas palavras tendem a se repetir nas pesquisas dentro de um determinado campo de estudo. Este fato está comprovado por intermédio da figura 04, quando fica nítido o fato de que algumas palavras aparecem de forma mais repetitiva nos trabalhos selecionados.

Conforme abordado na introdução e justificativa, alguns autores afirmam que o tema deve suscitar um forte relacionamento entre os governos e a sociedade. Para verificar se este fato está presente nos trabalhos, ou seja, se estes contextos são focos dos trabalhos, demonstra-se por intermédio da figura 04 qual o contexto (governo, sociedade ou ambos) foi foco principal dos trabalhos desenvolvidos.

Figura 04 - Contexto foco dos trabalhos sobre Dados Abertos

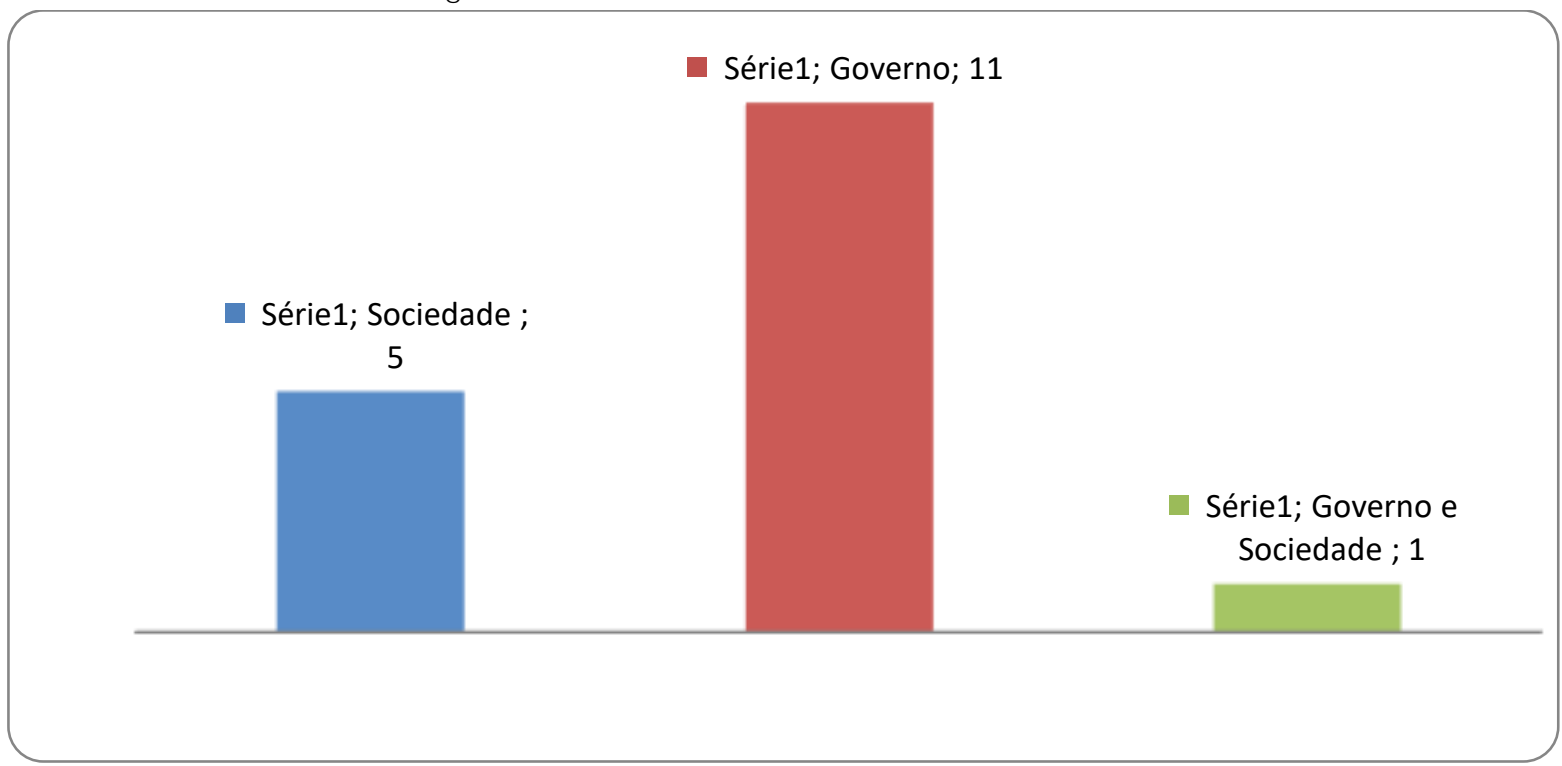

Fonte: Elaborado pelos autores (2018).

Sublinha-se que o foco dos estudos produzidos sobre dados abertos está, predominantemente, voltado para o contexto governo. De outra parte um número menor de trabalhos abordou o tema com foco na sociedade e um número bastante reduzido de trabalhos abordou ambos os focos. Assim, é possível sugerir que mais trabalhos sejam realizados tendo como foco o contexto sociedade, ou até mesmo ambos os contextos.

Com relação ao método adotado pelos pesquisadores, podemos destacar que o estudo de caso foi o método mais empregado, representando o total de $42 \%$ do total de trabalhos publicados. Com $24 \%$ dos trabalhos o método bibliográfico foi o segundo método mais adotado nas pesquisas.

\section{CONSIDERAÇÕES FINAIS}

0 trabalho atendeu a questão de pesquisa, sendo possível identificar que os trabalhos têm foco em governo e sociedade, e até mesmo existem trabalhos que focam em ambos os contextos. Este fato confirma a citação a diversos autores, que identificam que são necessários estudos e maior interação entre estes segmentos para que o tema tenha maior efetividade.

Com relação aos demais objetivos, estes também foram atendidos, pois ao demonstrar: autores, instituições, foco dos trabalhos, método empregado, rede de cooperação entre autores e instituições, palavras-chave mais utilizadas, demonstrou-se o panorama de desenvolvimento destes trabalhos no cenário brasileiro.

O tema é precoce no cenário acadêmico brasileiro, pois os trabalhos encontrados foram publicados após 2010, embora a busca tenha considerado como período inicial o ano de 2006. Por outro lado, é visível o crescimento do tema, pois o número de publicações cresce anualmente. Conclui-se que há avanço 
quantitativo de estudos neste campo, sendo registrado crescimento de publicações a cada ano, até o ano de 2015. Percebe-se com relação à abordagem metodológica emprega nas pesquisas o método estudo de caso com predominância.

Reconhece-se como fator limitador a quantidade de trabalhos selecionados. Sugere-se para futuros trabalhos, um levantamento que alcance um maior número de trabalhos, além de outros escopos de produções acadêmicas como dissertações e teses para obter-se uma maior base para a análise. Aconselha-se também, analisar outros aspectos da produção cientifica, como referências mais citadas, possibilitando assim identificar os trabalhos e autores que mais influenciam o tema.

\section{REFERÊNCIAS}

ALBANO, C. S. Dados Governamentais Abertos: Proposta de um modelo de produção e utilização de informações sob a ótica conceitual da cadeia de valor. Tese (Doutorado). 2014. Faculdade de Economia, Administração e Contabilidade da Universidade de São Paulo, São Paulo, 2014. Disponível em: http://www.teses.usp.br/teses/disponiveis/12/12139/tde-03062014-170642/pt-br.php. 2014. Acesso em: 13 mar. 2016.

ALBANO, C. S.; REINHARD, N. Desafios para governos e sociedade no ecossistema brasileiro de dados governamentais abertos (DGA). Cadernos Gestão Pública e Cidadania, v. 20, n. 67, p. 214-234, 2015.

AMARAL, R. M. et al. Uma visão da produção científica nos Anais do Encontro Nacional de Engenharia de Produção através da Bibliometria. In:ENCONTRO NACIONAL DE ENGENHARIA DE PRODUÇÃO, 24., 2004, Florianópolis. Anais [...]. Florianópolis: ENANPAD, 2004.

\section{ANGÉLICO, F. Lei de Acesso à Informação Pública e seus possíveis desdobramentos para a} accountability democrática no Brasil. 2012. Dissertação (Mestrado) - Escola de Administração de Empresas de São Paulo, Fundação Getúlio Vargas. 2012. Disponível em:

http://bibliotecadigital.fgv.br/dspace/handle/10438/9905. 2012. Acesso em: 10 set. 2016.

BRAGA, L. V.; GOMES, R. C. Governo Eletrônico e seu relacionamento com o desenvolvimento econômico e humano: Um estudo comparativo internacional. Revista do Serviço Público, v. 66, n. 4, p. 523-556, 2015.

BRASIL. Declaração de Governo Aberto. [2011]. Disponível em: http://www.governoaberto.cgu.gov.br/central-de-conteudo/documentos/arquivos/declaracao-governoaberto.pdf. 2011. Acesso em: 15 maio 2106.

CLABO, N. RAMOS-VIELBA, I. Reutilización de Datos Abiertos em la Administración Pública em España y uso de Licencias-Tipo. Revista Española de Documentación Científica, v. 39, n. 3, e097, 2015.

DELOITTE L. L. P. Open Data: Driving growth, ingenuity and innovation. London: Deloitte ToucheTohmatsuLimited. [2017]. Disponível em: https://www2.deloitte.com/content/dam/Deloitte/uk/Documents/deloitte-analytics/open-data-drivinggrowth-ingenuity-and-innovation.pdf. 2012. Acesso em: 20 abr. 2017.

FREITAS, R. K. V.; DACORSO, A. L. R. Inovação aberta na gestão pública: análise do plano de ação brasileiro para a Open Government Partnership. Revista Administração Pública, Rio de J aneiro, v. 48, n. 4, p. 869-888, 2014.

GUEDES, V.; BORSCHIVER, S. Bibliometria: uma ferramenta estatística para a gestão da informação e do conhecimento, em sistemas de informação, de comunicação e de avaliação científica e tecnológica. In: ENCONTRO NACIONAL DE CIÊNCIA DA INFORMAÇÃO, 6., 2005, Salvador. Anais [...]. Salvador, BA:UFBA, 2005.

GIL, A. C. Métodos e Técnicas de Pesquisa Social. 6. ed. São Paulo: Atlas, 2008. 
GURSTEIN, M. Open data: Empowering the empowered or effective data use for everyone? [2011].

Disponível em: http://firstmonday.org/ojs/index.php/fm/article/view/3316/2764. 2011. Acesso em: 20 set. 2016.

HARRISON, T. M.; PARDO, T. A.; COOK, M. Creating Open Government Ecosystems: A Research and Development Agenda. Future.Internet, v. 4, p. 900-928, 2012.

HeLLBERG, A. Policy, Process, People and Public Data. [2014]. Disponível em: https://link.springer.com/chapter/10.1007/978-3-662-44426-9_22. Acesso em: 10 mar. 2019.

JANSSEN, M.; CHARALABIDIS, Y.; ZUIDERWIJK, A. Benefits, Adoption Barriers and Myths of Open Data and Open Government. Information Systems Management, v. 2, n. 4, p. 258-268, 2012.

JOHNSON, J. A. From open data to information justice. Ethics and Information Technology, v. 16, n. 4, p. 263-274, 2014.

KLEIN, R. H.; LUCIANO, E.M.; MACADAR, M. A. Grau de transparência de dados abertos governamentais do site dados.rs.gov.br. E\&G Economia e Gestão, Belo Horizonte, v. 15, n. 41, p. 256-285, 2015.

LATHROP, D., RUMA, L. Open Government: Collaboration, Transparency, and Parcipation in Practice O'Peilly Media: Sebastopol, CA, USA. 2010.

MCKINSEY, C. GI. Open data: Unlocking innovation and performance with liquid information. [2013]. Disponível em: http://www.mckinsey.com/Insights/MGl/Research/Technology and Innovation. Acesso em: 10 jul. 2016.

MIGUEL, P. A. C. Metodologia de Pesquisa em Engenharia de Produção d Gestão de Operações. 2. ed. Rio de Janeiro: Elsevier, 2012.

MORAES, G.H. S. M.; MEIRELLES, F. S. Adoção de Governo Eletrônico no Brasil: A perspectiva do usuário do programa Nota Fiscal Paulista. In: ENCONTRO NACIONAL DE ENGENHARIA DE PRODUÇÃO, 38., 2014, Rio de Janeiro. Anais [...]. Rio de Janeiro: ANPA, 2014.

OLIVERIO, M. A. Governo Aberto como Ferramenta de Comunicação Entre o Governo e o Cidadão. In: CONGRESSO BRASILEIRO DE CIÊNCIAS DE COMUNICAÇÃO, 34., 2011, Recife. Anais [...]. Recife: Sociedade Brasileira de Estudos Interdisciplinares da Comunicação, 2011.

ONU. Global e-government read inessreport 2004: Towards access for opportunity. Nova York: ONU, 2004.

PARYCEK, P.; HOCHTL, J.; GINNER, M. Open Government Data Implementation Evaluation. J. theor.appl. electron. commer. res., Talca, v. 9, n. 2, p. 80-99, 2014.

PRINCE, A.; OLÍAS, L.; BRYS, C. Análisis de la cadena de valor del ecosistema de Datos Abiertos de la Ciudad de Buenos Aires. [2013]. Disponível em: http://www.princeconsulting.biz/pdf/7.pdf. 2013. Acesso em: 17 maio 2016.

SANTOS NETO, A. L. et al. Tecnologias de dados abertos para interligar bibliotecas, arquivos e museus: um caso machadiano. TransInformação, v. 25, n. 1, p. 81-87, 2013.

SAPENA, A. F.; PESET, F.; BENAVENT, R. A. Acesso a Los Datos Públicos y su Reutulización: opendata y open government. El profesional de lainformación, v. 20, n. 3, p. 259-268, 2011.

SPLITTER, K.; ROSA, C. A. Genealogia dos trabalhos bibliométricos em contabilidade. In:CONGRESSO USP DE CONTROLADORIA E CONTABILIDADE, 12., 2012, São Paulo. Anais [...]. São Paulo: USP, 2012. 
SPLITTER, K.; ROSA, C. A. R.; BORBA, J. A. Uma Análise das Características dos Trabalhos "Ditos" Bibliométricos Publicados no Enanpad entre 2000 e 2011. In:ENCONTRO NACIONAL DE ENGENHARIA DA PRODUÇÃO, 36., 2012, Rio de Janeiro. Anais [...]. Rio de Janeiro: ANPAD, 2012.

UBALDI, B. Open Government Data: Towards Empirical Analysis of Open Government Data Initiatives, OECD Working Papers on Public Governance. 2013. Disponível em: http://www.oecdilibrary.org/governance/open-government-data_5k46bj4f03s7-en. Acesso em: 15 jun. 2017.

ROBINSON, D.; ZELLER, W.; YU, D. Government data and the invisible hand. Yale J ournal of Law and Technology, v. 11, n. 160, artigo 4, 2009.

W3C. Manual dos Dados Abertos: governo. [2011]. Disponível em:

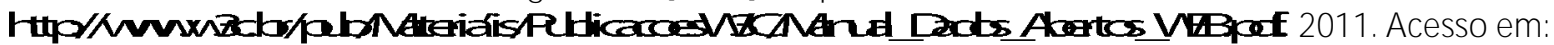
16 maio 2016. 